\title{
A Review of Hip Fracture Mortality-Why and How Does Such a Large Proportion of These Elderly Patients Die?
}

\author{
Aodhnait S. Fahy ${ }^{1}$, Fabian Wong ${ }^{1}$, Kumarapathan Kunasingam ${ }^{1}$, Daniel Neen ${ }^{1}$, \\ Frances Dockery ${ }^{2}$, Adil Ajuied', Diane L. Back ${ }^{1}$ \\ ${ }^{1}$ Trauma and Orthopaedics Unit, London, UK \\ 2Department of Ageing \& Health, Guy's and St Thomas' NHS Trust, London, UK \\ Email: fahy.aodhnait@mayo.edu
}

Received 11 March 2014; revised 10 April 2014; accepted 17 April 2014

Copyright (C) 2014 by authors and Scientific Research Publishing Inc.

This work is licensed under the Creative Commons Attribution International License (CC BY).

http://creativecommons.org/licenses/by/4.0/

c) (i) Open Access

\section{Abstract}

As the number of aged patients presenting with hip fractures continues to rise [1], the substantial mortality associated with this pathology must continue to be an area of scrutiny and improvement. While some dismiss the high mortality as an inevitable consequence of the comorbidities inherent in the original injury and the patient population, data suggest that at 2 years post-hip fracture, survivors' risks have regressed significantly and in fact, return to the same mortality risk as the non-fracture population [2]. This indicates that the risk of death is largely created specifically by the hip fracture and its treatment per se rather than the comorbidities of the hip fracture population, and offers scope for the potential reduction of these causes of death if we can identify and manage them appropriately. At a time when the incidence of hip fracture worldwide is on a steady rise, this review considers why and how these older patients die, and whether our developing guidelines and continuing research is adequately addressing these causes of death.

\section{Keywords}

Hip Fracture, Mortality, Older People, Proximal Femoral Fracture, Postoperative

\section{Introduction}

Over 110,000 hip fractures occurred in the UK last year with the mean age of patients affected being 79 [1]. Over the 25-year period from 2008 to 2033, the percentage of the population in the UK over 60 is expected to

${ }^{*}$ Corresponding author. 
rise from $22 \%$ to $27 \%$ with a numerical increase of $52 \%$ [2]. Epidemiological analysis suggests that hip fractures are likely to remain one of the leading causes of premature mortality amongst this population [3]. The mortality of this presentation is significant-with an estimated excess 30 day mortality of between 7 and $10 \%$, an excess 1 year mortality of between $18 \%$ and $35 \%$ [1] [4]. Addressing the underlying factors of the peri-and post-operative mortality appropriately and adequately will be vital to reducing the impact of this growing problem. The National Hip Fracture database (NHFD) established in 2007 in England was set up with this goal in mind, as there is clearly scope for improvement given the striking national variation in mortality rates, consequent possibly, on the equally striking national variation in peri-and post-operative management of hip fracture patients [1].

Surgical management of hip fractures-encompassing dynamic hip screw fixation, hemiarthroplasty, or total hip replacement-is the mainstay of treatment. Extensive analysis has been undertaken as to the causes of 30 day mortality and 1 year mortality after hip fracture surgery and further investigation has led to identification of the factors that place people at significantly higher risks. An autopsy-based review of people who died within 1 year of hip fracture surgery in 1995 indicated that pneumonia, cardiac failure, myocardial infarction and pulmonary embolism (PE) were the principal causes of death [4]. Other less common causes of mortality such as wound infection, urinary tract infection, gastrointestinal bleed and stroke made up the remainder [5]. While a considerable portion of the hip fracture conundrum also centres on post-operative morbidity and loss of independence, the breadth of the subject is such that these aspects should be analysed on another occasion. This review instead centres on the causes behind the excess mortality post-hip fracture, what factors are amenable to intervention and what is being or can be done to address these factors.

\section{What Causes Death Post-Hip Fracture?}

In the immediate post-operative phase, cardiac complications peak at 2 days, while pneumonia and PE peaked in the second week after injury and these complications significantly impact the in-hospital mortality [4]-[6]. For example, in patients who developed post-operative heart failure, mortality was $65 \%$ at 30 days and $92 \%$ at 1 year, while in patients who developed a post-operative chest infection, mortality at 30 days was $43 \%$ [5]. Identifying risk factors and interventions appropriate for these and other causes of post-hip fracture mortality will be key to reducing their impact.

\subsection{Cardiac Causes of Death and Targeted Interventions}

Cardiovascular disease is one of the most common co-morbid conditions in hip fracture patients as well as the most common cause of postoperative mortality [7]. Prompt cardiac triage can identify patients at high risk of peri-and post-operative cardiac complications. Silber et al. showed that while acute mortality is related to patient characteristics, later deaths are more closely related to hospital characteristics such as prompt cardiac treatment provision [8]. Pre-operative troponin T measurement has value identifying patients at higher risk of myocardial infarction and can predict cardiac complications in hip fracture patients, while postoperative analysis of troponin I further correlate with further increased mortality [8]. Some research on prevention of peri-operative cardiac mortality has focused on modulating sympathetic response with the peri-operative use of $\beta$-blockers [9]. Furthermore, epidural analgesia may exert a favourable effect on the stress response and pre-operative epidural analgesia specifically, was associated with fewer pre-operative cardiac events in hip fracture patients who had or were at risk of having coronary artery disease, in a randomised trial though event rate was low in this small study [10]. However it remains an area for further study in high risk patients.

A diagnosis of cardiac failure is associated with a two-fold higher risk of hip fracture [11], while new onset post-operative heart failure is a significant cause of mortality from hip fracture [5]. Age above 90yrs, male sex, a history of cardiovascular disease were all significant risks for developing post-operative heart failure [7] [8]. In patients who developed post-operative heart failure, mortality was $65 \%$ at 30 days and $92 \%$ at 1 year [5]. Assessment of baseline amino-terminal (NT) pro-BNP levels proved predictive of post-operative cardiac failure following hip fracture surgery [12], which was independent of pre-diagnosed congestive heart failure as a risk factor for peri-operative complications. Some groups have called for routine echocardiogram in all hip fracture patients pre-operatively-a potentially considerable resource burden which might also delay timely surgery though it may allow these high risk patients to be identified on admission [13]. No clinical trials have addressed the overall benefits of this approach. However, Mak’s systematic review which aimed to identify evidence-based guide- 
lines for the management of hip fractures in older persons, could not identify any consistent and specific interventions to reduce the heart failure-related burden [14].

\subsection{Respiratory Causes of Death and Targeted Interventions}

Pneumonia is an early and often remediable factor in post-operative mortality. Male sex, chronic obstructive pulmonary disease, enteral steroid use and greater age were all important risk factors for developing a chest infection after surgery for hip fracture [5], and those with multiple risk factors were significantly more at risk. Mortality from pneumonia was significantly less when surgery was performed within 24h of admission [15]. The significance held true when patients were control-matched for the significant comorbidities that can delay surgery while trying to optimise complex medical conditions [15]. The method of delivery of anaesthesia and analgesia may also be important in maintaining good respiratory function sufficient to avoid post-operative chest infections. A Cochrane review of regional anaesthesia for hip fractures showed regional anaesthesia to be associated with significantly decreased mortality at 1 month (6.9\%) [16]. Physiotherapy-driven lung expansion techniques such deep-breathing exercises and incentivised spirometry which have had success in reducing mortality in abdominal surgery should be given significant evaluation in proximal femoral fractures. Consideration could also be given to whether prophylactic antibiotics might be extended to provide cover for pneumonia in particularly high risk patients. A Cochrane Review of 21 studies addressing prophylactic antibiotics in hip fracture identified that most studies were poor to moderate [17], but pooled data suggested that prophylactic preoperative antibiotics reduced respiratory infections as well as wound infections. However this would need to be balanced against Clostridium difficile diarrhoea risk and selection of antibiotic resistance and warrants further study on risk/benefit ratio.

Patients with hip fractures are at inordinately high risk for the development of venous thromboembolism (VTE) and massive PE was the fourth highest cause of post-hip fracture mortality [4] [5]. Advanced age, surgical delay and prolonged surgery contribute significantly. Randomised controlled trials (RCTs) have shown that in the absence of VTE prophylaxis, total and proximal deep vein thrombosis (DVT) rates are approximately 50\% and $27 \%$ respectively, but with modern treatment strategies, the incidence can be reduced to as low as $1 \%$ [18]. Mortality from PE was significantly less when surgery was performed within 24 hours of admission [4] [15]. The benefit of early ambulation to prevent VTE further justifies aggressive surgical intervention. A systematic review of five clinical trials of mechanical prophylaxis of VTE post-hip fracture concluded that although the rate of DVT was reduced with these devices, all the studies were small, and there is no evidence that they protect against fatal PE or reduce overall mortality [19]. At present, most major organisations advocate at least 28 days of post-operative chemical prophylaxis, unless contraindicated [1]. The most recent Cochrane review of VTE prophylaxis for hip fracture includes 31 trials and unfractionated heparin and LMWH were shown to be protective against VTE without increasing bleeding risk. However, some confusion reigns over whether reduction of DVT risk does actually correlate with reduction of fatal PE, or whether we're measuring the wrong outcomes in many of these analyses. In particular, no form of heparin has been proven to protect against fatal PE and the Cochrane review recommends additional research for comparison for that outcome [reviewed in 18]. The effect of anaesthesia on vasodilation in surgical patients has been investigated with reference to DVT/PE risk. Comparison between general and regional techniques, with additional venous distension resulting from the use of muscle relaxants, was analysed through a recent large ESCORTE study which indicated that the incidence of VTE was similar for general and spinal anaesthesia [19].

\section{Other Factors Associated with Increased Mortality Risk Post-Hip Fracture}

While women dominate in terms of incidence, the outcome is poorer for the one third of hip fracture patients who are male even when controlled for age, fracture site, the number of medications, and chronic comorbidities. Men are at high risk post-operative complications such as well as mortality, and this is consistent in most published series [5] [20]. This emphasises the need for prompt post-operative evaluation of medical complications and treatment of comorbid conditions in the male hip fracture patient.

A BMI of 20 or less increases the risk of death including a recently published study identifying underweight patients to be at increased risk of developing an adverse cardiac event after hip fracture. Correspondingly, nutritional intervention reduced post-operative complications and mortality after 4 months in one study but post-operative complications were not accounted for in mortality analysis, limiting the conclusion [21]. 
Aharonoff showed that while individual comorbidities increased mortality, the cumulative effect of multiple comorbidities had a more significant effect on mortality [22]. The highest standardised mortality ratios at 1-2y follow-up is for patients who were ASA class 3 or 4 indicating multiple significant synergistic comorbidities [22]. In line with this, polypharmacy and in particular the use of diuretics is associated with increased rates of pneumonia, heart failure, further falls injury despite potential confounders like heart failure and hypertension [22].

Maxwell's analysis over ten years in Nottingham identified independent predictors of mortality at 20d as age, sex, $>2$ comorbidities, MMSE of $<6$, low $\mathrm{Hb}$ and presence of malignant disease. These variables were subsequently incorporated into a risk score, the Nottingham Hip Fracture Score. The number of deaths observed at 30 days, and the number of deaths predicted by these multiple interactive comorbidities indicated good concordance [23].

Dementia, or cognitive decline as well as functional impairment increase the risk of death in a statistically significant manner [24]. These factors remained statistically significant when adjusting for other medical comorbidities and age. In particular, preoperative RDRS score (a cumulative score analysing cognitive impairment, independence, and activities of daily living) was associated with a higher mortality indicating the importance of preoperative cognitive state and independence in hip fracture patients [24]. Delirium is highly prevalent post-hip fracture and is associated with poor outcomes following hip fracture surgery [25]. Haloperidol was shown to reduce delirium risk in one study but a more recent failed to show any benefit from this or from use of a specific clinical tool to detect delirium early following admission [reviewed in 25].

A recent study investigating the association of routine blood markers with postoperative mortality in hip fracture, indicated that routine admission blood tests including high potassium, low haemoglobin and low albumin could be predictably associated with an increased risk of mortality [26]. Most markedly, elevated creatinine on admission had a 3-fold increased risk of mortality in this patient setting indicating that focus on coincident acute kidney injury or optimising renal function in the context of chronic renal disease may also be necessary to improve hip fracture related mortality [26]. The red cell distribution width (RDW), an automated measure of variability in the red blood cell size on full blood count (FBC) is also independently associated with increased risk of short- and long-term mortality following hip fracture [6].

\section{Which Hospital Settings Increase the Risk of Death Post-Hip Fracture?}

As mentioned above, the timing of surgery on a hip fracture and the impact of any delay on mortality outcome is crucial. Patients with hip fracture should undergo surgery as quickly as possible with a view to reduced rates of post-operative complications and reduced mortality [15]. However, for individual patients, this needs to be balanced with the risks of not optimising medical comorbidities. A systematic review concluded that operative delay beyond $48 \mathrm{~h}$ after admission may increase the odds of 30 day all-cause mortality by $41 \%$ and of one year all-cause mortality by $32 \%$, despite adjustment for pre-operative comorbidities that may delay surgery [15].

Further analysis has investigated for variance between community and academic hospitals and showed minimal difference between the treatment settings for mortality. No clear link was seen between management at larger centres versus smaller hospitals with lower admissions of patients with hip fractures [27]. However, reduced nursing staffing levels are associated with increased in-hospital mortality in the hip fracture patient setting [28]. A further large cohort study indicated that higher quality care defined by meeting specific quality of care criteria (systematic pain assessment, nutritional care etc.) was associated with substantially lowered 30 day mortality [29] indicating that in-hospital adjustments can still alter the large mortality associated with hip fracture. Interestingly, management led by a medical team versus that led by an orthopaedic team does not alter mortality though early geriatric medical intervention was effective at reducing in-hospital mortality [30].

\section{Current Guidelines in Hip Fracture Management}

Recently developed National Institute of Clinical Excellence (NICE) guideline on hip fracture care identified early surgery (within 48h), regional anaesthesia, optimal analgesia (including nerve blockade), surgeon experience, choice of surgical approaches and implants, and optimal rehabilitation, as priorities in treatment [31]. NICE recommends rapid optimisation as fit for surgery which it details as prompt identification and treatment of correctible comorbidities. Correctible comorbidities were identified by them as volume depletion, electrolyte imbalance, anaemia, anticoagulation, uncontrolled diabetes, uncontrolled heart failure, arrhythmia or ischemia 
and acute chest infection. It is recommended performing surgery on the day of, or the day after, admission. The recommendations include prompt physiotherapy assessment and mobilisation on 1 day post surgery, subsequent patient mobilisation once a day and regular physiotherapy review. NICE also identify a thorough orthogeriatric assessment and MDT reviews as priorities in care. Continuous review of the efficacy of these guidelines and dedicated research to address the many unanswered questions on optimal management of this condition are still needed.

The NHFD, through audit and feedback nationally, has seen substantial improvements in time to surgery and pressure ulcer acquisition rates amongst other outcome measures [1]. A reduction in mortality has yet to be seen but it is anticipated that this will follow, and highlights the impact of audit and maintaining standards in hospital care for patients following hip fracture.

\section{Conclusion}

Hip fracture continues to be significant cause of mortality amongst an aging population though much has been done nationally to help address this [1] [31]. Prompt identification of the patient and hospital factors that place patients with hip fractures at increased risk can increase the efficacy of triage and management to reduce mortality. Addressing correctible comorbidities as early as possible is essential, as well as identification of underlying risk factors and incipient conditions which predispose the particular patients to specific causes of peri-and post-operative mortality. However, as highlighted there are a number of common causes of death which still harbour room for improvement in management and warrant further research to help guide clinicians on the optimal care of the growing cohort of patients with hip fractures ahead.

\section{Funding}

No funding was received for this work

\section{Conflicts of Interest}

None declared

\section{References}

[1] National Hip Fracture Database (2012) www.nhfd.co.uk

[2] Richmond, J., Aharonoff, G.B., Zuckerman, J.D. and Koval, K.J.J (2003) Mortality Risk after Hip Fracture. Orthopaedics and Trauma, 17, 53-56.

[3] White, S.M. and Griffiths, R. (2011) Projected Incidence of Proximal Femoral Fracture in England: A Report from the NHS Hip Fracture Anaesthesia Network (HIPFAN). Injury, 42, 1230-1233. http://dx.doi.org/10.1016/j.injury.2010.11.010

[4] Perez, J.V., Warwick, D.J., Case, C.P. and Bannister, G.C. (1992) Death after Proximal Femoral Fracture-An Autopsy Study. Injury, 26, 237-240.

[5] Roche, J.J., Wenn, R.T., Sahota, O. and Moran, C.G. (2005) Effect of Comorbidities and Post-Operative Complications on Mortality after Hip Fracture in Elderly People: Prospective Observational Cohort Study. BMJ, 331, 1374. http://dx.doi.org/10.1136/bmj.38643.663843.55

[6] Garbharran, U., Chinthapalli, S., Hopper, I., George, M., Back, D.L. and Dockery, F. (2013) Red Cell Distribution Width Is an Independent Predictor of Mortality in Hip Fracture. Age Ageing, 42, 258-261. http://dx.doi.org/10.1093/ageing/afs176

[7] Myers, A.H., Robinson, E.G., Van Natta, M.L., Michelson, J.D., Collins, K. and Baker, S.P. (1991) Hip Fractures among the Elderly: Factors Associated with In-Hospital Mortality. American Journal of Epidemiology, 134, 11281137.

[8] Silber, J.H., Williams, S.V., Krakauer, H. and Schwartz, J.S. (1992) Hospital and Patient Characteristics Associated with Death after Surgery_A Study of Adverse Occurrence and Failure to Rescue. Medical Care, 30, 615-629. http://dx.doi.org/10.1097/00005650-199207000-00004

[9] Urban, M.K., Markoqitz, S.M., Gordon, M.A., Urquhart, B.L. and Kligfield, P. (2000) Post-Operative Prophylactic Administration of Beta-Adrenergic Blockers in Patients at Risk for Myocardial Ischemia. Anesthesia \& Analgesia, 90, 1257-1261. http://dx.doi.org/10.1097/00000539-200006000-00001

[10] Matot, I., Oppenheim-Eden, A., Ratrot, R., et al. (2003) Pre-Operative Cardiac Events in Elderly Patients with Hip 
Fracture Randomised to Epidural or Conventional Analgesia. Anaesthesiology, 98, 156-163. http://dx.doi.org/10.1097/00000542-200301000-00025

[11] Carbone, L., Buzkova, P., Fink, H.A., et al. (2010) Hip Fractures and Heart Failure: Findings from the Cardiovascular Health Study. European Heart Journal, 31, 77-84. http://dx.doi.org/10.1093/eurheartj/ehp483

[12] Oscarsson, A., Fredrikson, M., Sorliden, M., Anskar, S. and Eintrei, C. (2009) N-Terminal Fragment of Pro-B-Type Natriueretic Peptide Is A Predictor of Cardiac Events in High-Risk Patients Undergoing Acute Hip Fracture Surgery. British Journal of Anaesthesia, 103, 206-212. http://dx.doi.org/10.1093/bja/aep139

[13] Heyburn, G. and McBrien, M.E. (2012) Preoperative Echocardiography for Hip Fractures: Time to Make It A Standard of Care. Anaesthesia, 67, 1189-1193. http://dx.doi.org/10.1111/j.1365-2044.2012.07330.x

[14] Mak, J.C., Cameron, I.D. and March, L.M. (2010) National Health and Medical Research Council. Evidence-Based Guidelines for the Management of Hip Fractures in Older Persons: An Update. Medical Journal of Australia, 192, 37-41.

[15] Moja, L., Piatti, A., Pecoraro, V., et al. (2012) Timing Matters in Hip Fracture Surgery: Patients Operated within 48 Hours Have Better Outcomes. A Meta-Analysis and Meta-Regression of over 190,000 Patients. PLoS One, 7, Article ID: e46175. http://dx.doi.org/10.1371/journal.pone.0046175

[16] Parker, M.J., Handoll, H.H. and Griffiths, R. (2004) Anaesthesia for Hip Fracture Surgery in Adults. Cochrane Database of Systematic Reviews, 19, Article ID: CD000521.

[17] Gillespie, W.J. and Walenkamp, G.H. (2010) Antibiotic Prophylaxis for Surgery for Proximal Femoral and Other Closed Long Bone Fractures. Cochrane Database of Systematic Reviews, 17, Article ID: CD000244.

[18] Marsland, D., Mears, S.C. and Kates, S.L. (2010) Venous Thromboembolism Prophylaxis for Hip Fractures. Osteoporosis International, 21, S593-S604. http://dx.doi.org/10.1007/s00198-010-1403-2

[19] Rosencher, N., Vielpeau, C., Emmerich, J., Fagnani, F., Samama, C.M., ESCORTE Group (2005) Venous Thromboembolism and Mortality after Hip Fracture Surgery: The ESCORTE Study. Journal of Thrombosis and Haemostasis, 3, 2006-2014. http://dx.doi.org/10.1111/j.1538-7836.2005.01545.x

[20] Kannegaard, P.N., van der Mark, S., Eiken, P. and Abrahamsen, B. (2010) Excess Mortality in Men Compared with Women Following a Hip Fracture. National analysis of comedications, comorbidity and survival. Age and Ageing, 39, 203-29. http://dx.doi.org/10.1093/ageing/afp221

[21] Duncan, D.G., Beck, S.J., Hood, K. and Johansen, A. (2006) Using Dietetic Assistants to Improve the Outcome of Hip Fracture: A Randomised Controlled Trial of Nutritional Support in an Acute Trauma Ward. Age and Ageing, 35, 148-153. http://dx.doi.org/10.1093/ageing/afj011

[22] Aharonoff, G.B., Koval, K.J., Skovron, M.L. and Zuckerman, J.D. (1997) Hip Fractures in the Elderly: Predictors of One Year Mortality. Journal of Orthopaedic Trauma, 11, 162-165. http://dx.doi.org/10.1097/00005131-199704000-00004

[23] Maxwell, M.J., Moran, C.G. and Moppett, I.K. (2008) Development and Validation of A Pre-Operative Scoring System to Predict 30 day Mortality in Patients Undergoing Hip Fracture Surgery. British Journal of Anaesthesia, 101, 511-517. http://dx.doi.org/10.1093/bja/aen236

[24] Seitz, D.P., Adunuri, N., Gill, S.S. and Rochon, P.A. (2011) Prevalence of Dementia and Cognitive Impairment among Older Adults with Hip Fractures. Journal of the American Medical Directors Association, 12, 556-564. http://dx.doi.org/10.1093/bja/aen236

[25] Vochteloo, A.J.H., Moerman, S., van der Burg, B., et al. (2011) Delirium Risk Screening and Haloperidol Prophylaxis Program in Hip Fracture Patients Is a Helpful Tool in Identifying High-Risk Patients, but Does Not Reduce the Incidence of Delirium. BMC Geriatrics, 11, 39.

[26] Mosfeldt, M., Pedersen, O.B., Riis, T., et al. (2012) Value of Routine Blood Tests for Prediction of Mortality Risk in Hip Fracture Patients. Acta Orthopaedica, 83, 31-35. http://dx.doi.org/10.3109/17453674.2011.652883

[27] Sund, R. (2010) Modeling the Volume-Effectiveness Relationship in the Case of Hip Fracture Treatment in Finland. Clinical Orthopaedics and Related Research, 468, 2218-2223.

[28] Schilling, P., Goulet, J.A. and Dougherty, P.J. (2011) Do Higher Hospital-wide Nurse Staffing Levels Reduce In-hospital Mortality in Elderly Patients with Hip Fractures: A Pilot Study. Clinical Orthopaedics and Related Research, 469, 2932-2940. http://dx.doi.org/10.1007/s11999-011-1917-8

[29] Nielsen, K.A., Jensen, J.C., Jensen, C.M., et al. (2009) Quality of Care and 30 day Mortality among Patients with Hip Fractures: A Nationwide Cohort Study. BMC Health Services Research, 12, 186.

[30] Vidan, M., Serra, J.A., Moreno, C., Riquelme, G. and Ortiz, J. (2005) Efficacy of a Comprehensive Geriatric Intervention in Older Patients Hospitalized for Hip Fracture: A Randomized, Controlled Trial. Journal of the American Geriatrics Society, 53, 1476-1482. http://dx.doi.org/10.1111/j.1532-5415.2005.53466.x

[31] NICE Hip Fracture Guidelines (2011) http://guidance.nice.org.uk/CG124 